DRAFT VERSION OCTOBER 24, 2018

Preprint typeset using LTEX style emulateapj v. 08/22/09

\title{
INSTABILITY-DRIVEN EVOLUTION OF POLOIDAL MAGNETIC FIELDS IN RELATIVISTIC STARS
}

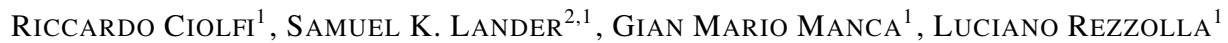 \\ Draft version October 24, 2018
}

\begin{abstract}
The problem of the stability of magnetic fields in stars has a long history and has been investigated in detail in perturbation theory. Here we consider the nonlinear evolution of a nonrotating neutron star with a purely poloidal magnetic field, in general relativity. We find that an instability develops in the region of the closed magnetic field lines and over an Alfvén timescale, as predicted by perturbation theory. After the initial unstable growth, our evolutions show that a toroidal magnetic field component is generated, which increases until it is locally comparable in strength with the poloidal one. On longer timescales the system relaxes to a new non-axisymmetric configuration with a reorganization of the stellar structure and large-amplitude oscillations, mostly in the fundamental mode. We discuss the energies involved in the instability and the impact they may have on the phenomenology of magnetar flares and on their detectability through gravitational-wave emission.

Subject headings: stars: neutron — gravitational waves — magnetohydrodynamics (MHD) — methods: numerical
\end{abstract}

\section{INTRODUCTION}

During at least two points within a neutron star's (NS) life, large-scale magnetic field rearrangement may occur. These are shortly after the formation of NSs in supernovae $(\overline{B o}-$ nanno et al. 2003), and also during the giant flares of magnetars (Thompson \& Duncan 1996, Geppert \& Rheinhardt 2006). Whilst similar rearrangements may also occur in other stars, they are likely to be particularly significant for the physics of NSs, where the fields are exceptionally strong: up to $10^{13} \mathrm{G}$ at the surface of normal pulsars and $10^{15} \mathrm{G}$ for magnetars. There are a variety of instabilities in NSs, and in a proto-NS magnetic fields may actually have a stabilising effect (see, e.g. Miralles et al. (2002); Bonanno et al. (2003)), but we are concerned here with the fast-acting "Tayler instability" which affects purely poloidal (or purely toroidal) magnetic fields in stars.

The magnetic-field geometry of a NS is important for the star's evolution, provides a distortion that may lead to gravitational radiation (Bonazzola \& Gourgoulhon 1996), as well as powering the mechanisms by which these stars may be observed: the pulsar emission for normal NSs, and the $\mathrm{X} / \gamma$-ray emission of magnetars. It is important therefore to determine which models of magnetised NSs are stable equilibria.

The study of magnetised stellar equilibria dates back to Chandrasekhar \& Fermi (1953). Since then, many possible magnetic equilibria have been studied, using both analytic and numerical techniques. These have included configurations with purely poloidal fields (Ferraro 1954; Monaghan 1965: Bocquet et al. 1995) and purely toroidal fields (Roxburgh 1963: Kiuchi \& Yoshida 2008), as well as mixed poloidaltoroidal configurations (Roxburgh 1966; Haskell et al. 2008 Tomimura \& Eriguchi 2005; Lander \& Jones 2009; Ciolfi et al. 2009, 2010).

However, constructing a configuration in equilibrium is only half the problem when modelling stellar magnetic fields; one also needs them to be stable over many dynamical timescales, since stellar magnetic fields have been observed

\footnotetext{
Electronic address: ciolfir@aei.mpg.de

${ }^{1}$ Max-Planck-Institut für Gravitationsphysik, Albert-Einstein-Institut, Potsdam, Germany

${ }^{2}$ School of Mathematics, University of Southampton, Southampton, UK
}

to be long-lived. This has proved to be a challenging problem for analytic methods, which can only study the initial localised instability and not the resultant field configuration. With purely poloidal and purely toroidal fields known to be unstable (Markey \& Tayler 1973; Wright 1973, Tayler 1973 Flowers \& Ruderman 1977), only mixed-field configurations are likely to exist in stars.

More recently it has become feasible to use numerical evolutions to study these hydromagnetic instabilities, with the benefit that the global behaviour of the instability may be studied (analytic works rely on local analyses), as well as the final outcome of the instability when the field undergoes significant rearrangement (Lander \& Jones 2011; Braithwaite 2007; Geppert \& Rheinhardt 2006, Kiuchi et al. 2011). Despite this recent progress, there are still very few models of stellar magnetic-field configurations whose stability has been assessed.

The instability-induced redistribution of magnetic flux is potentially a very violent event and it has been suggested as a trigger mechanism for the giant flares of magnetars (Thompson \& Duncan 1996). This redistribution is likely to be accompanied by a significant change to the mass quadrupole moment of a NS, making it a potentially detectable source of gravitational waves (GWs) (Kashiyama \& Ioka 2011; Corsi \& Owen 2011). For this reason it is important to understand the frequency, amplitude and duration these GWs may have.

This paper is organised as follows. In Section 2 we give a description of our computational infrastructure and initial stellar models. In Section 3 we present results from our evolutions, showing the generation of an instability and the subsequent reorganisation of the magnetic field into a more stable configuration. We also study the $\mathrm{GW}$ emission from the instability and assess its detectability. Conclusions are presented in Section 4.

\section{PHYSICAL SYSTEM AND NUMERICAL SETUP}

We model our initial NS as a nonrotating isolated fluid body in ideal magnetohydrodynamics (MHD) and with a purely poloidal magnetic field permeating it and extending to the exterior. The initial axisymmetric equilibrium configuration is generated by the LORENE code, which produces a fully relativistic solution and consistently accounts for the metric dis- 

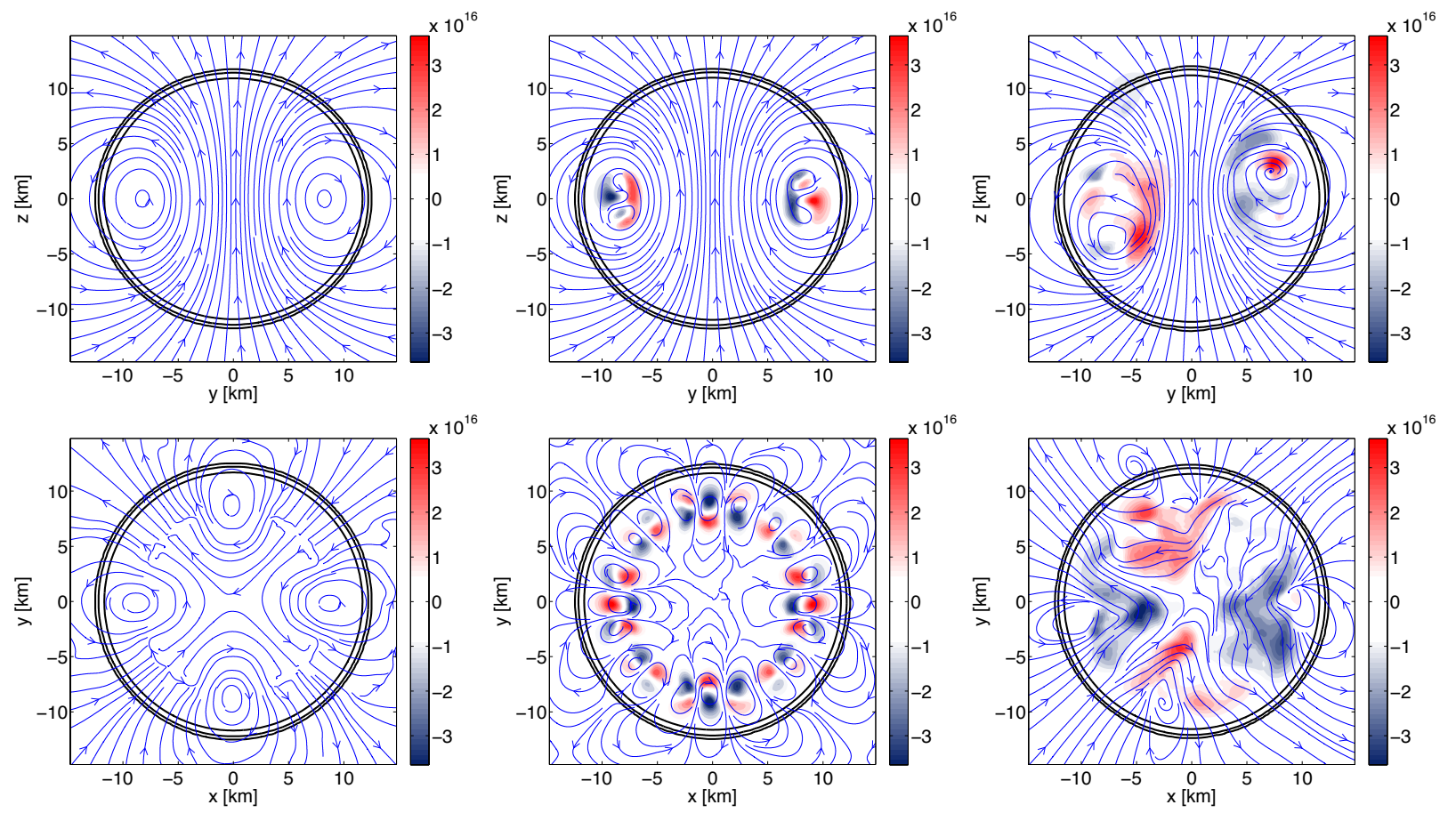

Figure 1. Snapshots of the development of the instability in our fiducial star, showing projections on the ( $x, z)$ plane (upper row) and ( $x, y)$ plane (lower row) of the simulation at times $t=1,3,10 \mathrm{~ms}$ (left to right), respectively. Shown with vector lines are the (global) magnetic-field lines, while the colours show the intensity of the toroidal magnetic field only; also reported are the iso-density contours of the rest-mass density near the stellar surface.

tortions and structure deformations induced by the magnetic field (see Bocquet et al. (1995)). The star is modelled as a polytrope with equation of state $p \equiv K \rho^{\Gamma}$, where $\Gamma=2$ and $K=98.5$, so that a NS with mass of $1.41 M_{\odot}$ has a radius of about $12.1 \mathrm{~km}$. Because the timescale for the instability is shorter (and computationally feasible) for stronger magnetic fields, we have considered stars with $B_{0} / 10^{16} \mathrm{G} \in[2.5,10]$, where $B_{0}$ is the value at the magnetic pole, selecting a value of $B_{0}=6.5 \times 10^{16} \mathrm{G}$ as the reference one.

Using these initial configurations, we perform generalrelativistic MHD simulations in three spatial dimensions under the Cowling approximation, i.e. we do not evolve the Einstein equations but consistently solve for the MHD equations in a fixed and curved spacetime. This choice is motivated by wanting to reduce computational costs and by the fact that the changes in the spacetime are expected to be intrinsically small. As a consequence, the GW emission is computed using the Newtonian quadrupole formula (Nagar et al. 2007; Baiotti et al. 2009). The evolutions are performed with the WhiskyMHD code, whose properties have been tested and discussed in a number of earlier papers (Giacomazzo \& Rezzolla 2007, Pollney et al. 2007; Giacomazzo et al. 2009, Giacomazzo et al. 2011). Our standard numerical setup consists of a grid with three refinement levels (Schnetter et al. 2004), the highest one having a resolution $h / M_{\odot}=0.17 \simeq 250 \mathrm{~m}$ and covering all of the star. The outer boundaries are placed at a distance of $54 M_{\odot} \simeq 79 \mathrm{~km}$.

The most salient difference of the code with respect to the references above is in the treatment of the atmosphere. We recall that as customary in relativistic hydrodynamics using finite-volume methods, we surround the star with a lowdensity "atmosphere", whose dynamics is prescribed by suitable boundary conditions. More specifically, the rest-mass density there is set to a constant value, while the fluid ve- locity is reset to zero (see Baiotti et al. (2005) for details). Although this prescription works very well in hydrodynamic simulations, it becomes problematic in ideal MHD, since it prevents any evolution of the magnetic field in this region. For sufficiently strong magnetic fields this approach can rapidly lead to errors at the stellar surface, which prematurely terminate the simulations. To improve on our treatment of the atmosphere and allow for a dynamics of the magnetic field at surface and outside the star, we add a magnetic diffusivity term to the induction equation, which we write as $\partial_{t}\left(\tilde{B}^{i}\right)=\partial_{j}\left(\tilde{v}^{i} \tilde{B}^{j}-\tilde{v}^{j} \tilde{B}^{i}\right)+\eta \partial_{i} \partial^{i} \tilde{B}^{j}$, where $\eta$ is the scalar resistivity (see Giacomazzo \& Rezzolla (2007); Giacomazzo et al. (2011) for details on the implementation in ideal-MHD case). Because we want to retain the ideal-MHD behaviour in the stellar interior and allow for an evolution in the atmosphere, we set the resistivity to zero within the bulk of the star, letting it increase continuously to its atmospheric value starting from a low-density region near the stellar surface. More specifically, we set $\eta(\rho)=\eta_{0} f(\rho)$, where $\rho$ is the rest-mass density, $f(\rho)$ is the Fermi function and $\eta_{0}$ is a free parameter. We used a reference value of $\eta_{0} / M_{\odot}=0.12$, but we will discuss how results change for a lower value.

Again to reduce computational costs and because we are interested in the development of magnetic-field instabilities, we add a perturbation designed to trigger them. From the analysis of Markey \& Tayler (1973) we expect a $\theta$-component of the velocity near the neutral line to induce the fastest-growing instability. Hence, we introduce a perturbation of azimuthal index $m=2$ in this quantity, such that the relative change in the magnetic field is $10^{-3}$. We have verified that the results do not change qualitatively for other choices of the perturbation and that an instability develops even without a perturbation.

\section{RESULTS}


Before discussing the nonlinear development of the instability, it is useful to recall the predictions of the perturbative studies of Markey \& Tayler (1973) and Wright (1973) about its early growth. In particular, we expect that: (i) the instability should be localised in the closed-field line region around the neutral line (i.e. where the poloidal magnetic field vanishes); (ii) the instability should occur after about an Alfvén timescale $\tau_{A}$ (if $\tau_{A} \sim 2 R \sqrt{4 \pi\langle\rho\rangle} / B_{0}$, where $\langle\rho\rangle$ is the average rest-mass density and $R$ the stellar radius, $\tau_{A} \sim 3 \mathrm{~ms}$ for our fiducial model); (iii) the (exponential) growth rate of the instability should scale linearly with magnetic field strength. As we will discuss, all of these expectations are met.

Figure 1 provides snapshots of the development of the instability in our fiducial star, showing projections on the $(x, z)$ plane (upper row) and $(x, y)$ plane (lower row) of the simulation at times $t=1,3,10 \mathrm{~ms}$ (left to right), respectively. These correspond to early, mid and late stages of the evolution. Shown with vectors lines are the (global) magnetic-field lines, while the colors show the intensity of the toroidal magnetic field only; also reported are the iso-density contours of the rest-mass density near the stellar surface.

As expected, the instability develops around the neutral line (left column), rapidly generating a toroidal magnetic field in this region (middle column). The growth of this component continues until it reaches a comparable strength to the poloidal one. At this point, the growth proceeds much more slowly, and the magnetic field evolution is less dramatic (right column), as the star evolves towards a new equilibrium. As revealed by the different panels in Fig. 1 the development of the instability breaks the axisymmetry of the initial configuration, leading to a complex structure with high azimuthal wave numbers (the $m=10$ component is dominant in the middle column), which is eventually replaced by an $m=2$ geometry at later stages (right column). While in this complex evolution the (small) $m=4$ component is probably inherited from the Cartesian coordinate system, it is interesting that the high- $m$ modes develop despite the initial perturbation being an $m=2$ one. Note also that the toroidal magnetic field produced by the instability is concentrated in vortices (smaller at early times and larger at later times) and that it changes sign both on meridian planes (see upper row) and on the equatorial one (see lower row). This late-time structure is different from the typical (axisymmetric) twisted-torus discussed in previous works (Braithwaite 2009; Ciolfi et al. 2009, Lander \& Jones 2009). We believe that this loss of axisymmetry follows from the conservation of magnetic helicity in ideal MHD (Woltjer 1958). Since purely poloidal (or purely toroidal) magnetic fields have zero helicity and the latter has to be conserved during a transformation in the ideal-MHD limit, the generation of a vortex structure represents the natural way in which a newly-generated toroidal field will not violate the initial zerohelicity of the system.

A few additional remarks are worth making about Fig. 1 The first one is about the evolution of the magnetic field in the regions right outside the star, which is essentially controlled by the resistivity there. Although the reference value used, $\eta_{0} / M_{\odot}=0.12$, is rather high and responsible for a considerable decay of the magnetic field, it also allows for a smooth evolution and removes the development of the discontinuities which would appear in the ideal-MHD limit. As we will discuss later on, the qualitative behaviour of the instability is not affected by the value of the resistivity or by the initial magnetic field strength. The second remark is about the

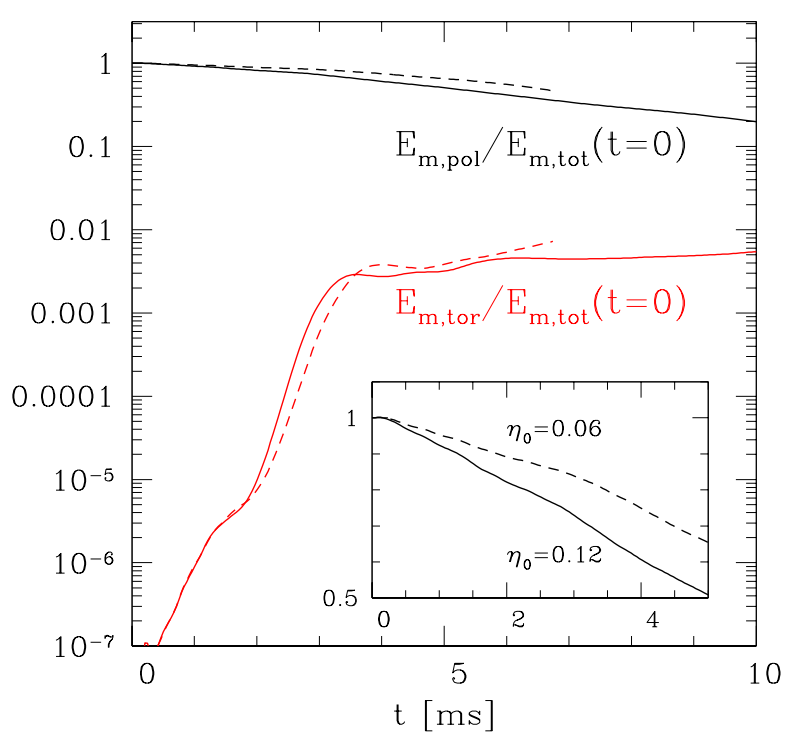

Figure 2. Evolution of the energy in the toroidal $E_{\mathrm{m} \text {,tor (red solid line) }}$ and poloidal $E_{\mathrm{m}, \text { pol }}$ components (black solid line), normalised to the initial value of the magnetic energy. The dashed lines refer to the corresponding evolution with $\eta_{0}=0.06$ and show a smaller dissipation of the magnetic field (see inset) but the same dynamics in the instability.

dynamics of the toroidal magnetic field that, as it grows to become locally comparable with the poloidal one, it also moves towards the stellar surface, where it can induce outflows of matter for smaller values of $\eta_{0}$. While a more detailed discussion of this process will be presented in a subsequent work, it is worth mentioning here that these winds could eject considerable amounts of matter $\left(\right.$ i.e. $\left.\sim 10^{-5}-10^{-4} M_{\odot}\right)$ and thus have direct connections with the magnetar phenomenology.

Figure 2 provides a more detailed analysis of the instability's behaviour, by monitoring the evolution of the energy in the toroidal $E_{\mathrm{m} \text {,tor }}$ (red solid line) and poloidal $E_{\mathrm{m}, \text { pol }} \mathrm{com}-$ ponents (black solid line), normalised to the initial value of the magnetic energy. Initially the toroidal component is very small, growing slowly until a time of $2 \mathrm{~ms}$. At this point, which is close to our Alfvén-timescale estimate of $3 \mathrm{~ms}$, there is a sudden exponential growth, which lasts for another $1 \mathrm{~ms}$. The exponential growth, which nicely matches the predictions of the linear-perturbation regime described by Markey $\&$ Tayler (1973), lasts for $\sim 0.5 \tau_{A}$ and then ceases, leaving a configuration which is seen to be roughly unchanged for several more Alfvén timescales. This suggests that the configuration shown in the right column of Fig. 1 is in a quasi equilibrium and is no longer susceptible to the instability. Note that whilst the local maxima of the field components are likely to dictate the system's stability, the energy eventually present in the toroidal-field component is only $\sim 3 \%$ of the total magnetic energy. This value may increase slightly on a much longer timescale.

Also reported in Fig. 2 as dashed lines are the corresponding evolution of the magnetic energies when a smaller resistivity of $\eta_{0} / M_{\odot}=0.06$ is used. Since the evolution of the instability in this case is qualitatively very similar ( $c f$. the evolution of $E_{\mathrm{m} \text {,tor }}$ ), we have confidence that our prescription for the resistive behaviour of the magnetic field near the stellar surface does not influence the dynamics of the instability. At the same time, however, a smaller resistivity is also re- 




Figure 3. Instability growth-rate as a function of the initial magnetic field strength for the NSs considered (red empty circles). Note the scaling remains approximately linear up to $B_{0} \simeq 7 \times 10^{16} \mathrm{G}$. Marked with a blue star is the corresponding growth-rate for $\eta_{0}=0.06$.

sponsible for a smaller decay of the poloidal magnetic field (see inset), which is considerably dissipated by the end of the simulation. While this behaviour is inevitable in a resistive context and has been reported also by other authors (Braithwaite 2007), it represents an aspect of these evolutions which could be improved with a fully consistent resistive MHD approach (Palenzuela et al. 2009).

Another important confirmation of the perturbative analysis is offered in Fig. 3, where we show the inverse of the growth-time $\tau$, defined through the exponential growth of the toroidal component, versus the initial magnetic-field strength (red empty circles). Note that the scaling is essentially linear for $B_{0} \lesssim 7 \times 10^{16} \mathrm{G}$, deviating from this for higher values, because of the stronger magnetic tension. More specifically, the stronger Lorentz force will tend to oppose the fluid motions in the polar direction near the neutral line and which trigger the instability. The presence of a linear scaling is essential to extend our results to typical pulsar magnetic-field strengths, thus estimating a growth-time of $\sim 10 \mathrm{~s}$ for a neutron star with $B_{0}=10^{12} \mathrm{G}$. Also marked in Fig. 3 (blue star) is the inverse growth-time for the fiducial star evolved with the smaller resistivity of $\eta_{0} / M_{\odot}=0.06$; again, the close similarity in the timescales confirms our expectation that the instability is not influenced by the choice of the resistivity.

The final discussion is reserved for the potential GW signal emitted during the development of the instability. In Fig. 4 we report the GW strain in the + and $\times$ polarizations as computed from the Newtonian quadrupole formula. It is quite apparent that the signal is not of a burst type but, rather, that the main effect of the instability is that of triggering largeamplitude oscillations of the star in its fundamental $F$-mode.

These GWs start emerging from the numerical noise already at $\sim 3.5 \mathrm{~ms}$, but are associated to high- $m$ oscillations and hence not efficient sources of GWs. However, as the magnetic field starts to approach the final $m=2$ configuration at $\sim 7 \mathrm{~ms}$, the oscillations become more efficient in producing a $\mathrm{GW}$ signal (Note that a $m=N$ pertubation

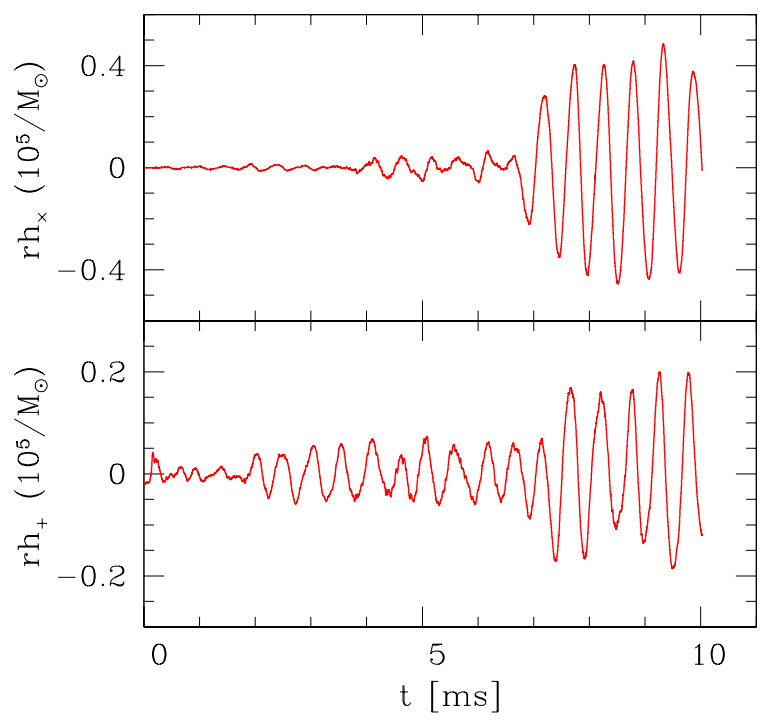

Figure 4. GW strain in the + and $\times$ polarizations. Note the instability triggers large-amplitude $F$-mode oscillations.

in the magnetic field leads to a $m=2 N$ perturbation in the density). Because these oscillations will have a rather narrow spectral distribution peaked around the $F$-mode frequency (which is not significantly affected by the presence of magnetic fields), they represent very good sources of a periodic signal, potentially detectable by future advanced detectors. Defining the root-sum-square amplitude of the cross polarization as $h_{\mathrm{rss}}=\left[\int_{-\infty}^{+\infty} d t h_{\times}^{2}(t)\right]^{1 / 2}$, and assuming that the oscillations will persist undamped for $\simeq 0.1-1 \mathrm{~s}$, we estimate $h_{\mathrm{rss}}=(0.54-1.7) \times 10^{-22}$ for a source at $10 \mathrm{kpc}$. The corresponding signal-to-noise ratio for a detector such as advanced-LIGO or advanced-Virgo is $S / N \simeq 1.6-5$, thus potentially observable. A more detailed analysis of the spectral properties of the GW signal will be presented in a future work. These waveforms represent the first estimate of the conversion of the kinetic energy generated through the instability into GWs. For weaker magnetic fields, perturbative analyses have suggested this coupling is much weaker (Levin \& van Hoven 2011), but more work is needed to investigate nonlinearly this regime.

\section{SUMMARY}

We report on numerical evolutions of the instability of poloidal magnetic fields in relativistic stars and the subsequent generation of a mixed-field configuration in quasiequilibrium. In agreement with the expectations from analytic perturbative studies (Markey \& Tayler 1973; Wright 1973), we show that the instability appears after about an Alfvén timescale, localised in the region of closed field lines. In addition, the growth-rate of the instability has the expected linear scaling with magnetic field up to very large field strengths.

The instability leads to the generation of a toroidal magnetic field, which starts with a complex and high- $m$ azimuthal structure, and is produced in local vortices. The instability ceases when the two field components are locally comparable in strength and the resulting configuration relaxes towards a geometry dominated by an $m=2$ toroidal component. This non-axisymmetric configuration is different from the simpler 
axisymmetric ones of earlier studies (Ciolfi et al. 2009, Lander \& Jones 2009, Braithwaite 2009; Ciolfi et al. 2010). However, it also represents the natural evolution of a system which is required to conserve its initial zero helicity in the idealMHD limit.

The total magnetic energy of the final configuration has around a $3 \%$ contribution from the toroidal component; this is comparable with the equilibria studied by Ciolfi et al. (2009) and Lander \& Jones (2009), but considerably lower than that of Braithwaite (2009), where stratified main-sequence stars were considered. Interestingly, the development of the instability also leads to the buoyancy of the newly-generated toroidal magnetic field and this can result into a mass outflow near the stellar surface. Additional work is needed to establish how these winds can be related to the phenomenology observed in giant flares of magnetars.

A consequence of the instability for the very strong magnetic fields considered here is that of triggering largeamplitude oscillations of the star in its $F$-mode. Our simulations show that the small-amplitude oscillations of the star are amplified by about an order of magnitude by the time the instability has saturated. Because these oscillations will have frequencies around the $F$-mode frequency, it is reasonable to perform searches for periodic signals associated to giant flares in magnetars. For a source at $10 \mathrm{kpc}$ with oscillations persisting undamped for $\simeq 0.1-1 \mathrm{~s}$, the root-sum-square amplitude at $1500 \mathrm{~Hz}$ will be $h_{\mathrm{rss}}=(0.54-1.7) \times 10^{-22}$, thus leading to a signal-to-noise ratio $S / N \simeq 1.6-5$ for a detector such as advanced-LIGO or advanced-Virgo.

During the completion of this work we have become aware of a very similar analysis carried out by Lasky et al. (2011), where the instability of purely poloidal magnetic fields in relativistic stars was also presented. Despite the different numerical setups, the results obtained by Lasky et al. (2011) about the development of the Tayler instability are in good agreement with those presented here, thus validating each other's conclusions.

We are grateful to Bruno Giacomazzo, Ian Jones and Yuri Levin for useful comments, and Roberto De Pietri for help in the GW estimates. Support comes also from the "Della Riccia" Foundation, from "CompStar", a Research Networking Programme of the European Science Foundation, and from the DFG grant SFB/Transregio 7.
REFERENCES

Baiotti, L., Bernuzzi, S., Corvino, G., De Pietri, R., \& Nagar, A. 2009, Phys. Rev. D, 79, 024002

Baiotti, L., Hawke, I., Montero, P. J., Löffler, F., Rezzolla, L., Stergioulas, N., Font, J. A., \& Seidel, E. 2005, Phys. Rev. D, 71, 024035

Bocquet, M., Bonazzola, S., Gourgoulhon, E., \& Novak, J. 1995, A\&A, 301, 757

Bonanno, A., Rezzolla, L., \& Urpin, V. 2003, Astron. Astrophys., 410, L33

Bonazzola, S., \& Gourgoulhon, E. 1996, A\&A, 312, 675

Braithwaite, J. 2007, A\&A, 469, 275

-. 2009, MNRAS, 397, 763

Chandrasekhar, S., \& Fermi, E. 1953, ApJ, 118, 116

Ciolfi, R., Ferrari, V., \& Gualtieri, L. 2010, MNRAS, 406, 2540

Ciolfi, R., Ferrari, V., Gualtieri, L., \& Pons, J. A. 2009, MNRAS, 397, 913

Corsi, A., \& Owen, B. J. 2011, Phys. Rev. D, 83, 104014

Ferraro, V. C. A. 1954, ApJ, 119, 407

Flowers, E., \& Ruderman, M. A. 1977, ApJ, 215, 302

Geppert, U., \& Rheinhardt, M. 2006, A\&A, 456, 639

Giacomazzo, B., \& Rezzolla, L. 2007, Class. Quantum Grav., 24, S235

Giacomazzo, B., Rezzolla, L., \& Baiotti, L. 2009, Mon. Not. R. Astron. Soc., 399, L164

Giacomazzo, B., Rezzolla, L., \& Baiotti, L. 2011, Phys. Rev. D, 83, 044014

Haskell, B., Samuelsson, L., Glampedakis, K., \& Andersson, N. 2008,

MNRAS, 385, 531

Kashiyama, K., \& Ioka, K. 2011, Phys. Rev. D, 83, 081302

Kiuchi, K., \& Yoshida, S. 2008, Phys. Rev. D, 78, 044045

Kiuchi, K., Yoshida, S., \& Shibata, M. 2011, ArXiv e-prints

Lander, S. K., \& Jones, D. I. 2009, MNRAS, 395, 2162

-. 2011, MNRAS, 412, 1730

Lasky, P. D., Zink, B., Kokkotas, K. D., \& Glampedakis, K. 2011, arXiv: 1105.1895

Levin, Y., \& van Hoven, M. 2011, arXiv:1103.0880

Markey, P., \& Tayler, R. J. 1973, MNRAS, 163, 77

Miralles, J. A., Pons, J. A., \& Urpin, V. A. 2002, ApJ, 574, 356

Monaghan, J. J. 1965, MNRAS, 131, 105

Nagar, A., Zanotti, O., Font, J. A., \& Rezzolla, L. 2007, Phys. Rev. D, 75, 044016

Palenzuela, C., Lehner, L., Reula, O., \& Rezzolla, L. 2009, Mon. Not. R. Astron. Soc., 394, 1727

Pollney, D., et al. 2007, Phys. Rev. D, 76, 124002

Roxburgh, I. W. 1963, MNRAS, 126, 67

- 1966, MNRAS, 132, 347

Schnetter, E., Hawley, S. H., \& Hawke, I. 2004, Class. Quantum Grav., 21, 1465

Tayler, R. J. 1973, MNRAS, 161, 365

Thompson, C., \& Duncan, R. C. 1996, ApJ, 473, 322

Tomimura, Y., \& Eriguchi, Y. 2005, MNRAS, 359, 1117

Woltjer, L. 1958, Proceedings of the National Academy of Science, 44, 833

Wright, G. A. E. 1973, MNRAS, 162, 339 